\title{
Raum- und Wissensgeschichte urbaner Problemzonen
}

\author{
Rezension zu Christiane Reinecke (2021): Die Ungleichheit der Städte. Urbane \\ Problemzonen im postkolonialen Frankreich und der Bundesrepublik. Göttingen: \\ Vandenhoeck \& Ruprecht
}

Anthony Miro Born

Abb. 1 Titel des Buches (Verlag Vandenhoeck \& Ruprecht)

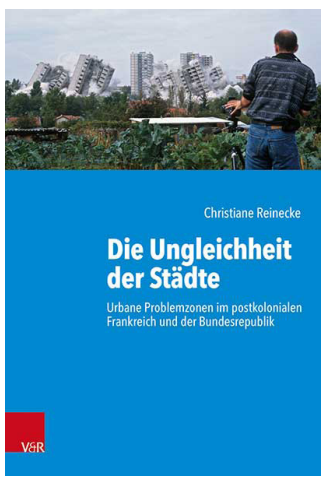

Es bedarf nur eines flüchtigen Blickes auf die andere Seite des Atlantiks, um den Einfluss sozialwissenschaftlicher Wissensproduktion auf Stadtentwicklungsprozesse herauszustellen. Seit Anfang der 1990er Jahre veranschaulicht das US-amerikanische HOPE-VI-Programm dort in bedrückendster Weise die Auswirkungen und Legitimationskraft fragwürdiger akademischer Konzepte wie culture of poverty, underclass oder concentrated poverty. Eine ganze Reihe angloamerikanischer Stadtforscher_innen hat dieses traurige Zusammenspiel unlängst treffend analysiert, allen voran der Historiker Michael B. Katz (2013).

In Bezug auf die deutschsprachige Stadtsoziologie und -geographie ist es gleichwohl bemerkenswert, wie selten der hiesige Mainstream die eigene Wirkmacht bislang reflektiert hat. Eine kritische Auseinandersetzung mit dem politischen und öffentlichen Einfluss der (nicht selten nordamerikanisch inspirierten) Narrative blieb bislang größtenteils aus. Dabei ist es durchaus angebracht zu fragen, welche Rolle etwa sozialwissenschaftliche Warnungen vor einer Ghetto-Apokalypse Ende der 1990er Jahre, Eigenlogik-Theorien, Nachbarschaftseffekt-Annahmen oder die jahrzehntelange Fetischisierung von abstrusen (aber oftmals „doppelten“) naturwissenschaftlichen Zyklusmodellen bei der Entwicklung urbaner Räume im deutschen Kontext hatten.Zwar wurde im Rahmen einer kritischen Auseinandersetzung mit der „Sozialen Stadt“ durchaus die Bedeutung der Stadtsoziologie bei der Ein- und Durchführung des Programms eruiert (siehe etwa die Debatten in German Politics \& Society 24/44), doch können die wenigen Ausnahmen kaum über die mangelnde Selbstreflexion der deutschsprachigen Stadtforschung hinwegsehen.

Gerade in diesem Kontext ist es überaus erfreulich, dass nun Christiane Reineckes herausragende Monografie Die Ungleichheit der Städte erschienen ist. Die kritische Stadtgeschichte analysiert, wie in Frankreich und Deutschland in der zweiten Hälfte des 20. Jahrhunderts mit sogenannten urbanen Problemzonen umgegangen wurde: Was (und wer) konstituierte diese sogenannten badlands, wie (und von wem) wurden sie gerahmt, gedeutet und verhandelt? Entstanden ist eine beeindruckende und vielschichtige Mischform aus Raum- und Wissensgeschichte; ein Buch, das man durchaus 
auch als Plädoyer an die Stadtforschungsdisziplin für mehr Selbstreflexion lesen kann. Denn so sind es neben Kommunalpolitiker_innen und Aktivist_innen ausgerechnet Sozialwissenschaftler_innen, die im Zentrum von Reineckes vergleichender Analyse stehen - und deren Rolle sich als weit mehr als nur die des_der außenstehenden Beobachter_in herausstellt.

Den Hauptteil des Buches bilden drei Kapitel, die sich verschiedenen Ausprägungen urbaner Marginalisierungsprozesse seit den 1950er Jahren widmen und vorherrschende Wissensbestände kritisch analysieren. Reinecke beginnt mit der „Blütezeit“ des französischen und westdeutschen Wohlfahrtsstaates und zeigt anhand der bidonvilles und der kommunalen Notunterkünfte, wie es beim Umgang mit städtischer Armut in den Nachkriegsjahrzehnten zunehmend zu einem Paradigmenwechsel von Disziplinierung und Separierung hin zu Aktivierung und Integration kam. Das zweite Kapitel befasst sich mit der symbolischen Abwertung von randstädtischen Großwohnsiedlungen. Mit besonderem Blick auf das Märkische Viertel in Berlin und auf Sarcelles in der nördlichen Peripherie von Paris zeichnet die Autorin nach, wie sich die Problematisierung dieser Siedlungen nur bedingt mit der Planungsgeschichte oder der Krise des Fordismus erklären lässt. Im dritten Kapitel widmet sich Reinecke schließlich explizit der Genealogie von Ghettoisierungs- und Segregationsnarrativen im westeuropäischen Kontext. Sie zeigt, wie bei der Verhandlung urbaner Ungleichheit das traditionelle Deutungsmuster der Klasse zunehmend „ethnisiert“ wurde.

Die Ungleichheit der Städte verleitet Lesende nicht nur zum verstärkten Nachdenken über die Wirkmacht der Wissensproduktion, sondern bricht gleichwohl mit einer ganzen Reihe beliebter Narrative innerhalb der heutigen Stadtforschung. So stehen beispielsweise Reineckes Beschreibungen der städtischen Exklusionspraktiken in den 1960er Jahren im Kontrast zu der immer noch anhaltenden glorifizierenden Pauschalisierung der fordistischkeynesianischen Ära in gegenwärtigen Stadtforschungsdiskursen. Wenngleich Peter Marcuse (1978) bereits in den 1970er Jahren vom „myth of the benevolent state“ schrieb, hat sich gerade im Kontext der Untersuchung neoliberaler Stadtentwicklung bedauerlicherweise vielerorts ein (zu) romantisches Bild der Nachkriegsjahrzehnte festgesetzt.[1] Wie so häufig in ihrem Buch bietet die Autorin eine differenziertere Erzählung, die ohne erhobenen Zeigefinger den Zwischentönen viel Raum gibt und gerade im französischen Kontext die postkolonialen Strukturen nie aus dem Blick verliert.

Überaus bemerkenswert ist zugleich Reineckes durchweg fesselnder Schreibstil. Das mag nicht zuletzt am Reichtum der Quellen liegen, die die Autorin für das Buch zusammengetragen und ausgewertet hat. (Neben akademischer Literatur und archivarischen Quellen wurde eine Vielzahl an Zeitungs- und Zeitschriftenartikeln sowie Film- und Fernsehsendungen analysiert.) Der westdeutsch-französische Vergleich wirkt zugleich nie zu schablonenhaft oder konstruiert. Vielmehr verdeutlicht das komparative Vorgehen geschickt die (häufig feinen) Unterschiede und Besonderheiten der beiden westeuropäischen Kontexte, ohne dabei jedoch in einen methodologischen Nationalismus zu verfallen. Reinecke ist überzeugt, dass sich hiesige Diskurse nicht ohne die Einbeziehung global zirkulierender Narrative verstehen lassen - insbesondere dem nordamerikanischen Kontext wird daher (zu Recht) ausreichend Platz eingeräumt. 
Einzig schade ist, dass Die Ungleichheit der Städte es vernachlässigt, sich differenzierter mit der Kategorie „Stadt“ und den damit verbundenen epistemologischen Annahmen auseinanderzusetzen. Reinecke erwähnt zwar, dass es schwieriger geworden sei, „Städtisches von Nicht-Städtischem abzugrenzen“ (Reinecke 2021: 15), doch wird ausgerechnet das Urbane an einigen Stellen als vorgegebene, selbstverständliche Realität und Form pauschalisiert. Daraus folgt nicht nur, dass das problematische Urban-ageNarrativ geradezu reproduziert wird - bereits in der Einleitung schreibt Reinecke: „Dabei lebte im 20. Jahrhundert infolge der fortschreitenden Urbanisierung erstmals der Großteil der europäischen Bevölkerung in Städten“(ebd.: 10 f.).Vielmehr versperrt sich so die Möglichkeit, verstärkter in die Analyse mit einzubeziehen, wie die untersuchten Akteur_innen auch das „Städtische“ selbst verschieden gerahmt und gedeutet haben. Insbesondere Neil Brenners und Christian Schmids (2014, 2015) epistemologische Kritik an urbanen Kategorien und Kategorisierungen hätte sich die Autorin hier an einigen Stellen gut zunutze machen können.

Nichtsdestotrotz bleibt Christiane Reineckes Monografie eine sehr beeindruckende und inspirierende Abhandlung - nicht zuletzt für diejenigen Forscher_innen, die sich mit urbanen Marginalisierungsprozessen, Großwohnsiedlungen und territorialer Stigmatisierung aus einer historischen Perspektive beschäftigen möchten. Gleichzeitig offeriert Die Ungleichheit der Städte auch allen anderen Stadtsoziolog_innen und -geograph_innen eine wertvolle Botschaft: die Wirkmacht akademischer Wissensproduktion nicht zu unterschätzen. Für viele kritische Stadtforscher_innen ist es zwar bereits Inbegriff guter Wissenschaft, darüber nachzudenken, inwiefern der akademische „Betrieb“ Wege für eine progressivere Stadt ebnen kann. Doch Reineckes historische Analyse mahnt in bemerkenswerter Weise, dass es trotz (und gerade wegen!) der betont guten Intentionen vielerorts unabdingbar bleibt, auch die (symbolische) Gewalt und politischen Konsequenzen akademischer Kategorisierungen und Klassifikationen explizit zu untersuchen und verstärkt zu reflektieren - nicht nur post festum.

„Manchmal bringen unsere Wissenschaften Arten von Menschen hervor, die es in gewisser Weise so zuvor nicht gab" (Reinecke 2021: 198), zitiert Reinecke treffend den kanadischen Philosophen Ian Hacking. Tatsächlich fällt es schwer, dabei nicht auch an gegenwärtige - vermeintlich gut gemeinte - Stadtforschungen zu denken.[2]

\section{Endnoten}

[1] Siehe etwa Wacquant (2009) für einige explizite Beispiele, für eine Kritik siehe Mayer (2010).

[2] Zu gut erinnere ich mich noch, wie in einer von mir besuchten Erstsemesterveranstaltung Bewohner_innen eines stigmatisierten Neubauviertels an mehreren Stellen des Lehrmaterials bemitleidenswert als die drei „A“ beschrieben wurden: „Alte, Arme, Arbeitslose“. Und erst kürzlich entdeckte ich eine ZDF-Reportage über Armut in Deutschland, in der auch ein Stadtsoziologe zur Rolle der Nachbarschaft kurz zu Wort kam. Der Forscher hatte vor allem drei Beobachtungen in einer westdeutschen Hochhaussiedlung machen können: „rauchende Eltern, die Kinderwägen schieben, Kampfhunde ohne Leine und exzessives Trinken in der Öffentlichkeit“. 


\section{Autor_innen}

Anthony Miro Born forscht zu urbanen Marginalisierungsprozessen, symbolischer Ungleichheit und sozialer Mobilität.

a.m.born@lse.ac.uk

\section{Literatur}

Brenner, Neil / Schmid, Christian (2014): The „Urban Age” in question. In: International Journal of Urban and Regional Research 38/3, 731-755.

Brenner, Neil / Schmid, Christian (2015): Towards a new epistemology of the urban? In: City 19/2-3, 151-182.

Katz, Michael B. (2013): The undeserving poor. America's enduring confrontation with poverty. Oxford: Oxford University Press.

Marcuse, Peter (1978): The myth of the benevolent state: towards a theory of housing. New York: Columbia University, Graduate School of Architecture and Planning.

Mayer, Margit (2010): Punishing the poor - a debate: Some questions on Wacquant's theorizing the neoliberal state. In: Theoretical Criminology 14/1: 93-103.

Reinecke, Christiane (2021): Die Ungleichheit der Städte. Urbane Problemzonen im postkolonialen Frankreich und der Bundesrepublik. Göttingen: Vandenhoeck \& Ruprecht.

Wacquant, Loï (2009): Punishing the poor. The neoliberal government of social insecurity. Durham, NC: Duke University Press. 